\title{
Gravitational waves propagating into Friedmann-Robertson-Walker universes
}

\author{
by Jiří Bičák* \\ Department of Theoretical Physics, Faculty of Mathematics and Physics, \\ Charles University, V Holešovičkách 2, 18000 Prague 8, Czech Republic \\ and Jerry B. Griffiths ${ }^{\dagger}$ \\ Department of Mathematical Sciences, Loughborough University, \\ Loughborough, Leicestershire. LE11 3TU, U.K.
}

August 1, 2018

\begin{abstract}
We consider space-times with two isometries which represent gravitational waves with distinct wavefronts which propagate into exact Friedmann-Robertson-Walker (FRW) universes. The geometry of possible wavefronts is analysed in detail in all three types of FRW models. In the spatially flat and open universes, the wavefronts can be planar or cylindrical; in the closed case they are toroidal. Exact solutions are given which describe gravitational waves propagating into the FRW universes with a fluid with a stiff equation of state. It is shown that the plane-fronted waves may include impulsive or step (shock) components, while the cylindrical waves in the spatially flat and open universes and the toroidal waves in closed universes may contain steps. In general, wavefronts may exist which have an arbitrary finite degree of smoothness. In all cases, the waves are backscattered. The head-on collision of such waves is also briefly mentioned.
\end{abstract}

${ }^{*}$ E-mail: bicak@hp03.troja.mff.cuni.cz

${ }^{\dagger} \mathrm{E}-$ mail: J.B.Griffiths@Lboro.ac.uk 


\section{Introduction}

Most work on gravitational waves in cosmological backgrounds has been based on first order approximation methods. However, if gravitational radiation played a significant role in the universe, then the waves would cause the symmetries of the standard homogeneous and isotropic models to be violated. In order to treat general, large perturbations one has to turn to a numerical approach. However, when space-time is assumed to have two spacelike Killing vectors, exact solutions containing gravitational waves or solitons can be constructed, at least for vacuum or for scalar or electromagnetic fields or for a stiff perfect fluid in which the pressure is equal to the density. Some of these solutions have been reviewed for example by Carmeli et al [1], by Adams et al [2], and recently by Verdaguer [3].

In previous work on gravitational waves in cosmological backgrounds, the exact solutions considered have been interpreted as perturbations extending over whole universes. As such, it has not been possible to give an analysis of the geometry of the wave surfaces. In this paper we present the new class of solutions representing gravitational waves propagating into homogeneous, isotropic universes. The space-time ahead of a gravitational wave is taken to be an exact Friedmann-Robertson-Walker (FRW) universe. Since this has spatial sections of constant curvature, the geometrical properties of the wavefront can be explicitly determined. For these solutions, we can also analyse the character of the wavefront: whether it contains a $\delta$-function impulse, or a shock (step) wavefront, or is smooth in the sense that the first $n$ derivatives of the Weyl tensor are continuous across the front.

We consider space-times which admit two commuting, hypersurface orthogonal spacelike Killing vectors. These can be described by the line element

$$
d s^{2}=e^{-M}\left(d \eta^{2}-d \mu^{2}\right)-e^{-U}\left(e^{V} d x^{2}+e^{-V} d y^{2}\right),
$$

where the metric functions $U, V$ and $M$ depend on the coordinates $\eta$ and $\mu$ only. In fact, all the FRW models can also be written in this form for a perfect fluid with any equation of state, with or without a cosmological constant. Thus, certain exact solutions described by this line element can be considered to be (large) perturbations of these FRW models, provided they reduce to particular models in appropriate limits. (Other classes of inhomogeneous universes which can reproduce the FRW models in certain limits have recently been reviewed by Krasiński [四.)

It may immediately be noticed that, in the form of the line element (1.1), two families of null hypersurfaces, $\eta \pm \mu=$ const., are singled out in a natural way. These surfaces may be regarded as wave surfaces for both approximate and exact gravitational waves. The geometry of these wavefronts in the FRW universes of all three types will be analysed in detail in this paper. These null hypersurfaces may also be considered as suitable wavefronts for some other radiative fields.

In a series of recent papers [5]-[8], some families of exact solutions have been outlined which describe the propagation (and collision) of gravitational waves into FRW backgrounds in the above form. In these solutions, the space-time is exactly FRW with stiff 
fluid ahead of the waves. Such waves therefore have well-defined wavefronts. It was previously noted that these waves are necessarily backscattered, and that their interactions following head-on collisions do not induce additional singularities into the space-time. However, complete metrics were not given and the structure of the wavefront was not analysed. A method for obtaining complete solutions was later described in [9].

It is the purpose of this paper to complete the derivation of the solutions representing waves with distinct wavefronts propagating into FRW backgrounds, and to summarise them from a unified point of view for each possible type of wavefront and all three types of FRW models. We will describe the waves by considering the most general form of the solution of the main field equation for a distinct wavefront. Using the method outlined in [9], we will complete the integration of the subsidiary equations, thus determining the whole metric. We will also analyse the structure of the wavefront in each case. It will be shown that these may include impulsive components in some cases, but may contain step (or shock) waves in all cases. Waves in which the Weyl tensor has $n$ continuous derivatives are also discussed.

We will first describe the geometrical character of possible wavefronts in each FRW model in order to treat the properties of all the above solutions from a unified point of view. In Sections 2-4, we will consider the spatially flat, open and closed FRW models in turn. It will be shown that, in the spatially flat and open FRW universes, the gravitational waves can have plane wavefronts. In these universes, the gravitational wavefronts can alternatively be cylindrical. In the case of the closed FRW universe, the wavefronts are toroidal. In the Appendix, we describe in detail how a 2-torus, forming a wavefront, can be constructed in the closed model. The character of the wavefronts reflects only the symmetries assumed. This approach is thus relevant also in any study of more general waves and cosmological models than those treated here. It may be of interest to analyse even small (linear) perturbations of FRW universes with these symmetries, with other equations of state and, possibly, with a non-vanishing cosmological constant.

After summarizing the properties of the FRW universes with a stiff fluid in Section 5, we study in Section 6 the general family of solutions which describe waves with distinct wavefronts propagating into these backgrounds. Then, in Sections $7-11$, we analyse respectively the separate cases of plane and cylindrical waves in spatially flat and open universes, and toroidal waves in a closed universe.

As well as analysing the character of the wavefront, we also find that, for the waves with plane wavefronts the only space-time singularity is that describing the big bang. However, our cylindrical and toroidal waves will, in general, propagate away from (and be backscattered towards) a singular axis, which can be considered as both "source" and "absorber" of the waves. Other remarks appropriate by way of conclusion are collected in Section 12 . 


\section{Wavefronts in spatially flat FRW universes}

In the spatially flat case (with curvature index $k=0$ ), the Robertson-Walker line element may be written in either of the forms

$$
\begin{aligned}
& d s^{2}=d t^{2}-R^{2}(t)\left(d x^{2}+d y^{2}+d z^{2}\right), \\
& d s^{2}=d t^{2}-R^{2}(t)\left(d \rho^{2}+\rho^{2} d \phi^{2}+d z^{2}\right),
\end{aligned}
$$

where $R(t)$ is determined by Friedmann's equation. In order to consider null hypersurfaces, it is convenient to introduce the "conformal time" coordinate $\eta$ such that

$$
d t=R(\eta) d \eta
$$

The line elements (2.1) and (2.2) can then be written in the form (1.1):

$$
\begin{aligned}
& d s^{2}=R^{2}(\eta)\left(d \eta^{2}-d z^{2}\right)-R^{2}(\eta)\left(d x^{2}+d y^{2}\right), \\
& d s^{2}=R^{2}(\eta)\left(d \eta^{2}-d \rho^{2}\right)-R^{2}(\eta)\left(\rho^{2} d \phi^{2}+d z^{2}\right) .
\end{aligned}
$$

In (2.4), surfaces on which $\eta=$ const., $z=$ const. are planes, and the null hypersurfaces $\eta-z=$ const. represent plane wavefronts. Clearly, plane wave surfaces oriented in any direction can immediately be constructed. Alternatively, in the form (2.5), surfaces on which $\eta=$ const., $\rho=$ const. are cylinders, and the null hypersurfaces $\eta-\rho=$ const. represent expanding cylindrical wavefronts.

\section{$3 \quad$ Wavefronts in FRW open universes}

The standard form of the metric of an open FRW model with negative spatial curvature $(k=-1)$ in coordinates $\chi \in[0,+\infty), \theta \in[0, \pi), \phi \in[0,2 \pi)$ reads

$$
d s^{2}=d t^{2}-R^{2}(t)\left[d \chi^{2}+\sinh ^{2} \chi\left(\mathrm{d} \theta^{2}+\sin ^{2} \theta d \phi^{2}\right)\right] .
$$

As is well known [10], the spatial 3-geometry on a hypersurface $t=t_{0}=$ const.,

$$
d \ell^{2}=R_{0}^{2}\left[d \chi^{2}+\sinh ^{2} \chi\left(d \theta^{2}+\sin ^{2} \theta d \phi^{2}\right)\right],
$$

where $R_{0}=R\left(t_{0}\right)$, can be represented as 3-dimensional hyperboloid

$$
V^{2}-X^{2}-Y^{2}-Z^{2}=R_{0}^{2},
$$

embedded in a 4-dimensional Minkowski space with Lorentzian coordinates $V, X, Y, Z$, where

$$
\begin{aligned}
V & =R_{0} \cosh \chi, \\
Z & =R_{0} \sinh \chi \cos \theta, \\
X & =R_{0} \sinh \chi \sin \theta \cos \phi, \\
Y & =R_{0} \sinh \chi \sin \theta \sin \phi .
\end{aligned}
$$


In order to find 2-planes in the open FRW model, we introduce alternative coordinates in which the 3 -metric is conformally flat. By putting

$$
\begin{aligned}
X & =R_{0} \frac{x}{z}, \quad Y=R_{0} \frac{y}{z}, \\
Z & =\frac{R_{0}}{2 z}\left(1-x^{2}-y^{2}-z^{2}\right), \\
V & =\frac{R_{0}}{2 z}\left(1+x^{2}+y^{2}+z^{2}\right),
\end{aligned}
$$

where $x, y \in(-\infty, \infty), z \in(0,+\infty)$, we find (3.3) to be satisfied. These coordinates sweep out the whole "positive" $(V>0)$ sheet of the hyperboloid (3.3). The 3-metric now takes the form

$$
d \ell^{2}=\frac{R_{0}^{2}}{z^{2}}\left(d x^{2}+d y^{2}+d z^{2}\right)
$$

Clearly, putting $z=$ const. we obtain 2 -surfaces with intrinsically flat geometry. Both the 3-metric (3.6) and the whole space-time metric admit, on these 2-surfaces, not only the translational Killing vectors $\partial_{x}$ and $\partial_{y}$, but also the rotational Killing vector $y \partial_{x}-x \partial_{y}$. The proper lengths along the whole orbits of both $\partial_{x}$ and $\partial_{y}$ are infinite. Thus, these 2 -surfaces on which $z=$ const. can be considered to be 2-planes.

In figure 1 we illustrate these 2-planes in the embedding diagram of the section $Y=0$, or $\phi=0$ and $\pi$, through the 3-hyperboloid (3.3) in a 3-dimensional Minkowski space with coordinates $V, X, Z$. Since $z=z_{0}=$ const. implies $V+Z=R_{0} / z_{0}=$ const., the 2-planes are formed by cutting the 3-hyperboloid (3.3) along "null hyperplanes" $V+Z=$ const. in the Minkowski space. In this way we obtain the 2-planes described in coordinates $V, X, Y, Z$ by

$$
\begin{aligned}
-Z & =\frac{1}{2} z_{0}\left(X^{2}+Y^{2}\right)+Z_{0}, \\
Z_{0} & =\frac{1}{2} z_{0} R_{0}^{2}-z_{0}^{-1}=\text { const. } \\
V & =R_{0} z_{0}^{-1}-Z .
\end{aligned}
$$

For a fixed $z_{0}$, this is a 2-paraboloid which in figure 1 reduces to a parabola, the $Y$ dimension having been suppressed. (Figure 1 remains the same after replacing $X$ by $Y$.) On the other hand, by considering sections of Minkowski space on which $Z=$ const., equations (3.5) and (3.7) for fixed $z=z_{0}$ imply $X^{2}+Y^{2}=$ const., or $x^{2}+y^{2}=$ const. With these cuts of the 3 -hyperboloid, the infinity of the 2-planes cannot be seen. Indeed, in terms of the original coordinates $\chi, \theta, \phi$, the condition $V+Z=R_{0} z_{0}{ }^{-1}$ reads $\cosh \chi+$ $\sinh \chi \cos \theta=z_{0}{ }^{-1}$. In order to consider $\chi \rightarrow \infty$, we have to let $\theta \rightarrow \pi$, as expected for paraboloids with axes in that direction. In choosing coordinates according to (3.5), we "singled out" the coordinate $z$; since the 3-hyperboloid is isotropic and homogeneous, we can construct other sets of 2-planes by simple transformations.

Cylindrical 2-surfaces in the open FRW models can easily be found by introducing coordinates $\rho \in[0,+\infty), y \in(-\infty,+\infty), \phi \in[0,2 \pi)$ such that

$$
\begin{aligned}
V & =R_{0} \cosh \rho \cosh y, \\
Z & =R_{0} \cosh \rho \sinh y, \\
X & =R_{0} \sinh \rho \cos \phi, \\
Y & =R_{0} \sinh \rho \sin \phi .
\end{aligned}
$$


The 3-metric on the 3-hyperboloid then takes the form

$$
d \ell^{2}=R_{0}^{2}\left[d \rho^{2}+\sinh ^{2} \rho d \phi^{2}+\cosh ^{2} \rho d y^{2}\right]
$$

Putting $\rho=\rho_{0}=$ const., we obtain cylindrical 2 -surfaces. The proper length along the orbits of the Killing vector $\partial_{\phi}$ is finite, being $2 \pi R_{0} \sinh \rho$, but it is infinite along the orbits of $\partial_{y}$. Although the local geometry on the cylinder is intrinsically flat, there exists no other Killing vector in this 2-space as occurs in the case of the 2-plane.

In figure 2, a cylindrical 2-surface $\rho=\rho_{0}$ is illustrated in the same embedding diagram as in figure 1. From (3.8), we now find

$$
\begin{aligned}
X^{2}+Y^{2} & =\left(R_{0} \sinh \rho_{0}\right)^{2}=\text { const. } \\
V^{2}-Z^{2} & =\left(R_{0} \cosh \rho_{0}\right)^{2}=\text { const. }
\end{aligned}
$$

Since the $Y$-dimension is suppressed $\left(\phi=0\right.$ and $\pi$ ) in figure 2, we obtain $X= \pm R_{0} \sinh \rho_{0}$ and $V^{2}-Z^{2}=\left(R_{0} \cosh \rho_{0}\right)^{2}$. These are two hyperbolae, representing two generators of the cylindrical 2 -surface $\rho=\rho_{0}$. The axis of the cylinder is given by $\rho=0$, i.e. $\theta=0$ or $\pi$, or $X=Y=0$. Considering the section of the 3-hyperboloid (3.3) on which $Z=0$, the 2-cylinder would be represented by the circle $X^{2}+Y^{2}=\left(R_{0} \sinh \rho_{0}\right)^{2}, V=R_{0} \cosh \rho_{0}$, as follows from (3.10). Clearly, for $\rho_{0}$ small, the 2-cylinder would approximate to an ordinary cylinder in $E^{3}$.

In terms of the two new sets of coordinates (3.5) and (3.8), the line element (3.1) takes the following forms

$$
\begin{aligned}
d s^{2} & =d t^{2}-R^{2}(t)\left[\frac{1}{z^{2}}\left(d x^{2}+d y^{2}+d z^{2}\right)\right] \\
d s^{2} & =d t^{2}-R^{2}(t)\left[d \rho^{2}+\sinh ^{2} \rho d \phi^{2}+\cosh ^{2} \rho d y^{2}\right] .
\end{aligned}
$$

Introducing the new coordinate

$$
\mu=\log z
$$

the line element (3.11) becomes

$$
d s^{2}=d t^{2}-R^{2}(t)\left[d \mu^{2}+e^{-2 \mu}\left(d x^{2}+d y^{2}\right)\right] .
$$

Finally, going over to the "conformal time" coordinate $\eta$ in both cases, we arrive at the metric

$$
d s^{2}=R^{2}(\eta)\left(d \eta^{2}-d \mu^{2}\right)-R^{2}(\eta) e^{-2 \mu}\left(d x^{2}+d y^{2}\right) .
$$

Alternatively, the line element (3.12) can be written as

$$
d s^{2}=R^{2}(\eta)\left(d \eta^{2}-d \rho^{2}\right)-\frac{1}{2} R^{2}(\eta) \sinh 2 \rho\left(\tanh \rho d \phi^{2}+\operatorname{coth} \rho d y^{2}\right) .
$$

Both metrics (3.15) and 3.16) are now expressed in the form (1.1). 


\section{Wavefronts in FRW closed universes}

We start with the standard form of the metric for a closed FRW model having positive spatial curvature $(k=1)$

$$
d s^{2}=d t^{2}-R^{2}(t)\left[d \chi^{2}+\sin ^{2} \chi\left(d \theta^{2}+\sin ^{2} \theta d \phi^{2}\right)\right],
$$

where $\chi \in[0, \pi], \theta \in[0, \pi], \phi \in[0,2 \pi]$. It is well known that the spatial 3-geometry on a hypersurface of homogeneity $t=t_{0}=$ const.,

$$
d \ell^{2}=R_{0}^{2}\left[d \chi^{2}+\sin ^{2} \chi\left(d \theta^{2}+\sin ^{2} \theta d \phi^{2}\right)\right]
$$

where $R_{0}=R\left(t_{0}\right)$, can be visualised as a 3 -dimensional sphere

$$
W^{2}+X^{2}+Y^{2}+Z^{2}=R_{0}^{2}
$$

embedded in a 4-dimensional Euclidean space with cartesian coordinates $W, X, Y, Z$. By setting

$$
\begin{aligned}
W & =R_{0} \cos \chi \\
Z & =R_{0} \sin \chi \cos \theta, \\
X & =R_{0} \sin \chi \sin \theta \cos \phi \\
Y & =R_{0} \sin \chi \sin \theta \sin \phi
\end{aligned}
$$

Eq. (4.3) is satisfied.

In the Appendix it is demonstrated in detail that, with the symmetries assumed, a typical wave surface in a closed FRW model is a 2-torus in the 3 -sphere. This is best described by parametrizing the whole 3 -sphere by coordinates $\zeta \in[0, \pi / 2], \sigma \in[0,2 \pi]$, $\delta \in[0,2 \pi]$ such that (cf. Eq. (A.5) in the Appendix)

$$
\begin{aligned}
W & =R_{0} \cos \zeta \cos \sigma \\
Z & =R_{0} \cos \zeta \sin \sigma \\
X & =R_{0} \sin \zeta \cos \delta \\
Y & =R_{0} \sin \zeta \sin \delta
\end{aligned}
$$

The 2-torus is specified by the choice of the parameter $\kappa=\cos \zeta$. (For an illustration, see Figure 8 in the Appendix.)

In terms of the new coordinates, the line element (4.1) takes the form

$$
d s^{2}=d t^{2}-R^{2}(t)\left[d \zeta^{2}+\sin ^{2} \zeta d \delta^{2}+\cos ^{2} \zeta d \sigma^{2}\right]
$$

or, using the conformal time $\eta$,

$$
d s^{2}=R^{2}(\eta)\left(d \eta^{2}-d \zeta^{2}\right)-\frac{1}{2} R^{2}(\eta) \sin 2 \zeta\left(\tan \zeta d \delta^{2}+\cot \zeta d \sigma^{2}\right),
$$

which is clearly in the form (1.1). 


\section{FRW universes with a stiff fluid}

We now consider the case of a "stiff" perfect fluid in which the pressure is equal to the density. This equation of state may be considered as a "modern version of aether" (see 畉) which enables mathematical relativists to generate a variety of new solutions. However, it may also have played an important role in the period of high densities after the big bang [11], 12], or even in the interior of cold condensed objects. Its role as a limiting equation of state of neutron matter was studied thoroughly in the full field theory by Walecka 13]. Difficult and important problems arise, of course, when studying gravitational waves which propagate in dust or in fluids with a more realistic equation of state. However, in the case of a stiff fluid, families of exact radiative solutions can be obtained which can be considered to be perturbations of the FRW models with a stiff fluid. In this section, we review the unperturbed background models.

The equation of state of the fluid is taken to be $\tilde{p}=\tilde{\rho}$. (We use tildes to denote quantities referring to the fluid to avoid confusion with the same symbols used elsewhere.) With this, the equation of energy conservation $\left(\tilde{\rho} R^{3}\right)_{,}=-\tilde{p}\left(R^{3}\right){ }_{t}$ implies that

$$
\tilde{\rho} R^{6}=\tilde{\rho}_{0} R_{0}^{6}=\text { const. }=\gamma_{0}=\frac{3}{8 \pi} \gamma^{2},
$$

where the dimension of the constant $\gamma$ is (length) $)^{2}$. The standard Friedmann equation, $R,{ }_{t}^{2}=-k+\gamma^{2} R^{-4}, k=0, \pm 1$, can then easily be integrated after introducing the conformal time coordinate $\eta$ in accordance with (2.3). The solutions read [14], [7]

$$
\begin{array}{rlrl}
R & =[\gamma \sinh 2 \eta]^{\frac{1}{2}}, & \text { if } k=-1, \\
R=[\gamma 2 \eta]^{\frac{1}{2}}, & \text { if } k=0, \\
R=[\gamma \sin 2 \eta]^{\frac{1}{2}}, & \text { if } k=+1 .
\end{array}
$$

In [7] the physical time $t$ is expressed explicitly in terms of $\eta$ by means of elliptic integrals in the cases $k= \pm 1$. For the spatially flat case one gets simply

$$
R=(3 \gamma t)^{\frac{1}{3}},
$$

so that the flat FRW universe with a stiff fluid expands slower than the corresponding universe with dust for which $R \sim t^{\frac{2}{3}}$ (see e.g. [10]). For the open universe, one finds [0] $R \cong t$ at large times, i.e. exactly the same asymptote as the standard open Friedmann universes with dust or radiation. All these open universes thus approach the flat Milne universe, for which $R=t$ exactly.

The "gravitational role" of the pressure in slowing down the expansion in the spatially flat case can also be seen in the "speeding up" of the recollapse in the case of the closed FRW models. The conformal time $\eta$ is also often called the "arc parameter" since it determines the radius of arc distance on the 3 -sphere covered by a photon travelling since the start of the expansion [10]. In the case of the closed universe with dust, the range of $\eta$ is $2 \pi$ - a photon makes "one trip" around the whole universe before its final collapse. In the model with radiation, in which $\tilde{p}=\tilde{\rho} / 3$, the range of $\eta$ is $\pi$ - a photon only gets as far as the antipodal point of the universe. In the universe with a stiff fluid, the last equation 
in (5.2) shows that the range of $\eta$ is $\pi / 2$ - a photon succeeds in traveling only one quarter of the distance around the whole universe (say, from the pole to the equator). This will be relevant in Section 11 where we shall consider exact toroidal waves propagating into a closed FRW background with a stiff fluid.

It is easy to write the line element of all three types of FRW models in the form (1.1). Since $\gamma$ is an arbitrary constant with dimension (length) ${ }^{2}$, we may consider (1.1) in the dimensionless form $d s^{2} / \gamma^{2}$. Without loss of generality we may thus put $\gamma=1$. Comparing now (2.4), (2.5), (3.15), 3.16) and (4.7) with $R(\eta)$ substituted from (5.2) with the general form (1.1), we find the following:

Case 1a. In the spatially flat case with the plane 2-surfaces $z=$ const., we have $\mu=z \in$ $(-\infty,+\infty),(x, y) \in \mathbb{R}^{2}, \eta \in[0, \infty)$, and

$$
e^{-U}=2 \eta, \quad e^{-M}=2 \eta, \quad V=0 .
$$

Case 1b. In the spatially flat case with the cylindrical 2-surfaces $\rho=$ const., $\mu=\rho \in$ $[0, \infty), x=\phi \in[0,2 \pi), y=z \in(-\infty,+\infty), \eta \in[0, \infty)$, and

$$
e^{-U}=2 \eta \rho, \quad e^{-M}=2 \eta, \quad e^{V}=\rho .
$$

Case 2a. In the open model with the plane 2-surfaces $z=$ const. or $\mu=$ const., we have $\mu \in(-\infty,+\infty),(x, y) \in \mathbb{R}^{2}, \eta \in[0, \infty)$, and

$$
e^{-U}=\sinh 2 \eta e^{-2 \mu}, \quad e^{-M}=\sinh 2 \eta, \quad V=0 .
$$

Case 2b. In the open model with the cylindrical 2-surfaces $\rho=$ const., $\mu=\rho \in[0, \infty)$, $x=\phi \in[0,2 \pi), y=z \in(-\infty,+\infty), \eta \in[0, \infty)$, and

$$
e^{-U}=\sinh 2 \eta \sinh 2 \rho, \quad e^{-M}=\sinh 2 \eta, \quad e^{V}=\tanh \rho .
$$

Case 3. In the closed model with the toroidal 2-surfaces $\zeta=$ const., we have $\mu=\zeta \in$ $[0, \pi / 2], x=\delta \in[0,2 \pi), y=\sigma \in[0,2 \pi), \eta \in[0, \pi / 2]$, and

$$
e^{-U}=\sin 2 \eta \sin 2 \zeta, \quad e^{-M}=\sin 2 \eta, \quad e^{V}=\tan \zeta
$$

It is well known [15] that, in a space-time with the metric (1.1), a stiff perfect fluid can be associated with a scalar potential $\tilde{\sigma}(\eta, \mu)$ such that the density and 4-velocity of the fluid are given by

$$
16 \pi \tilde{\rho}=e^{M}\left(\tilde{\sigma}_{\eta}^{2}-\tilde{\sigma}_{\mu}^{2}\right), \quad \tilde{u}_{\alpha}=\tilde{\sigma}_{, \alpha} /\left(\tilde{\sigma}_{, \beta} \tilde{\sigma}^{, \beta}\right)^{1 / 2},
$$

and, as a consequence of Einstein's equations, the fluid potential $\tilde{\sigma}$ satisfies

$$
\tilde{\sigma}_{\eta \eta}-U_{\eta} \tilde{\sigma}_{\eta}-\tilde{\sigma}_{\mu \mu}+U_{\mu} \tilde{\sigma}_{\mu}=0
$$


For the above FRW universes with a stiff fluid, the expressions for $\tilde{\sigma}$ are found to be

$$
\begin{array}{lll}
\tilde{\sigma}=3^{1 / 2} \log \eta & \text { for } & k=0, \\
\tilde{\sigma}=3^{1 / 2} \log \tanh \eta & \text { for } & k=-1, \\
\tilde{\sigma}=3^{1 / 2} \log \tan \eta & \text { for } & k=+1 .
\end{array}
$$

In the spatially flat and open cases, it should be noted that these expressions for $\tilde{\sigma}$ apply both in the plane and cylindrical cases. Clearly, all coordinate systems above are comoving with the fluid.

Using these background metric functions, we now turn to the construction of the waves which propagate into these backgrounds.

\section{Waves in FRW universes with a stiff fluid}

We return to the general line-element (1.1). In the case of a stiff perfect fluid, and vanishing cosmological constant, Einstein's field equations imply that $e^{-U}$ satisfies the wave equation

$$
\left(e^{-U}\right)_{\eta \eta}-\left(e^{-U}\right)_{\mu \mu}=0
$$

and $V$ satisfies the linear equation

$$
V_{\eta \eta}-U_{\eta} V_{\eta}-V_{\mu \mu}+U_{\mu} V_{\mu}=0 .
$$

It may immediately be noted that, in general, Eq. (6.1) can be integrated to give

$$
e^{-U}=f(\eta-\mu)+g(\eta+\mu)
$$

where $f$ and $g$ are arbitrary functions. In many cases, it is convenient to adopt $f$ and $g$ as coordinates, at least in some region of space-time in which they are not constant. The arbitrariness thus retained can be used to describe families of exact solutions. It can be seen, however, that singularities of some type will occur when $f+g=0$. In addition, in a closed universe, $f$ and $g$ cannot be monotonic functions over the entire space-time, and it is then more convenient to revert to coordinates that are more directly related to $\eta$ and $\mu$.

Taking $f$ and $g$ as coordinates, equations (6.2) and (5.10) take the form of EulerPoisson-Darboux equations with non-integer coefficients

$$
\begin{aligned}
& (f+g) V_{f g}+\frac{1}{2} V_{f}+\frac{1}{2} V_{g}=0, \\
& (f+g) \tilde{\sigma}_{f g}+\frac{1}{2} \tilde{\sigma}_{f}+\frac{1}{2} \tilde{\sigma}_{g}=0 .
\end{aligned}
$$

For any solutions of these equations, the remaining metric function $M$ can be found by quadratures. Putting

$$
e^{-M}=\frac{f^{\prime} g^{\prime}}{(f+g)^{1 / 2}} e^{-S}
$$

the remaining field equations become

$$
S_{f}=-\frac{1}{2}(f+g)\left(V_{f}^{2}+\tilde{\sigma}_{f}^{2}\right), \quad S_{g}=-\frac{1}{2}(f+g)\left(V_{g}^{2}+\tilde{\sigma}_{g}^{2}\right),
$$


which are automatically integrable in view of (6.4) and (6.5). However, these equations are also significant in determining conditions on the derivatives of $V$ and $\sigma$ when considering junction conditions across possible wavefronts. In addition, care has to be taken on any hypersurface on which $f^{\prime}$ or $g^{\prime}$ are zero. Using a null tetrad system that is naturally adapted to the coordinates (with the null vectors given by $\ell_{\alpha}=2^{-1 / 2} e^{-M / 2}\left(\eta_{, \alpha}-\mu_{\alpha}\right)$ and $n_{\alpha}=2^{-1 / 2} e^{-M / 2}\left(\eta, \alpha+\mu_{\alpha}\right)$, the non-zero components of the Weyl tensor are given in terms of the coordinates $f$ and $g$ by

$$
\begin{aligned}
& \Psi_{0}=-\frac{1}{2} g^{\prime 2} e^{M}\left(2 V_{g g}+\frac{3}{(f+g)} V_{g}-(f+g) V_{g}\left(V_{g}^{2}+\tilde{\sigma}_{g}^{2}\right)\right), \\
& \Psi_{2}=-\frac{1}{6} f^{\prime} g^{\prime} e^{M}\left(\frac{3}{(f+g)^{2}}-3 V_{f} V_{g}-\tilde{\sigma}_{f} \tilde{\sigma}_{g}\right), \\
& \Psi_{4}=-\frac{1}{2} f^{\prime 2} e^{M}\left(2 V_{f f}+\frac{3}{(f+g)} V_{f}-(f+g) V_{f}\left(V_{f}^{2}+\tilde{\sigma}_{f}^{2}\right)\right) .
\end{aligned}
$$

In many cases of interest, it is possible to choose $f$ such that $f=0$ represents a null hypersurface. On such a hypersurface, $\eta-\mu=$ const. It is then convenient to adopt a gauge such that $e^{-U}=f+g$ takes the same form on either side of $f=0$, thus facilitating the possible choice of $f$ and $g$ as coordinates. It may then be noticed that, since (6.4) is linear in $V$, we may superpose a background solution of (6.4) on either side of the hypersurface with another solution which is non-zero on one side only. In this way, we can construct an exact solution which contains a distinct wavefront which is given by the null hypersurface $f=0$. Of course, it is necessary to satisfy the appropriate (O'Brien-Synge) junction conditions [18] across the wavefront. In this case, these conditions require that both $V$ and $M$ be continuous across $f=0$. In the cases we are considering in this paper, it is found using Eqs. (6.7) that the continuity of $M$ implies that $V_{f}$ must also be bounded on $f=0$.

In the class of solutions we wish to consider, the background is taken in turns to be appropriate forms of the various FRW models with a stiff fluid given by (5.4)-(5.8). The additional component of $V$ can then be interpreted as introducing a purely gravitational wave. In addition, the expression for $\tilde{\sigma}$ is taken to be unchanged across the wavefront. This effectively eliminates the introduction of acoustic waves in the fluid of the type considered by Tabensky and Taub [19]. However, since Eq. (6.5) for $\tilde{\sigma}$ is identical to Eq. (6.4) for $V$, acoustic waves could also be considered by including the same kind of additional terms in $\tilde{\sigma}$. Of course, this equivalence occurs only for a stiff fluid in which the characteristics for both gravitational and acoustic waves are the same.

As introduced in [9], we now consider the appropriate class of self-similar solutions of (6.4) of the form

$$
V_{k}(f, g)=(f+g)^{k} H_{k}\left(\frac{g-f}{f+g}\right),
$$

where $k$ is an arbitrary real parameter whose range will be determined below, and the function $H_{k}(\zeta)$ is a solution of the equation

$$
\left(1-\zeta^{2}\right) H_{k}^{\prime \prime}+(2 k-1) \zeta H_{k}^{\prime}-k^{2} H_{k}=0
$$

This equation admits a class of solutions which can be expressed in terms of hypergeometric functions $F(a, b ; c ; z)$ such that the solutions (6.9) of (6.4) can be expressed in either of 
the forms

$$
\begin{aligned}
(f+g)^{k} H_{k}\left(\frac{g-f}{f+g}\right) & =c_{k} \frac{f^{k+1 / 2}}{g^{1 / 2}} F\left(\frac{1}{2}, 1+k ; \frac{3}{2}+k ;-\frac{f}{g}\right), \\
& =c_{k} \frac{f^{k+1 / 2}}{(f+g)^{1 / 2}} F\left(\frac{1}{2}, \frac{1}{2} ; \frac{3}{2}+k ; \frac{f}{f+g}\right),
\end{aligned}
$$

where $c_{k}=\frac{\Gamma(1 / 2)}{\Gamma(k+3 / 2)}$. Both forms of this expression (6.11) and (6.12) will be useful below, depending on the appropriate ranges of $f$ and $g$. For integer values of $k$, these functions take particularly simple forms in which successive functions $H_{k}(\zeta)$ can be obtained recursively using the relation

$$
H_{k}(\zeta)=\int_{1}^{\zeta} H_{k-1}\left(\zeta^{\prime}\right) d \zeta^{\prime}
$$

with the initial solution $H_{0}(\zeta)=\cos ^{-1} \zeta$ if $f \geq 0$, or $H_{0}(\zeta)=\cosh ^{-1} \zeta$ if $f \leq 0$. It may be noted that the scaling constants $c_{k}$ have been included in (6.11) and (6.12) to ensure that no additional scale factor appears in $(\sqrt[6.13]{ })$, and we may generally put $H_{k}^{\prime}(\zeta)=H_{k-1}(\zeta)$.

As can be seen from (6.11) or (6.12), the significant feature of the solution (6.9) is that $V=0$ and $V_{f}$ is bounded on the null hypersurface $f=0$ provided $k \geq \frac{1}{2}$. It may thus be matched across the null hypersurface $f=0$ to the solution $V=0$ on the other side. It then represents some kind of strong gravitational wave with wavefront $f=0$. As mentioned above, since (6.4) is linear, this solution may be added to some other background solution to represent the propagation of a gravitational wave in that background. For FRW stiff fluid backgrounds, the condition $k \geq \frac{1}{2}$ ensures that both $V$ and $M$ are continuous across the null hypersurface $f=0$, thus satisfying the O'Brien-Synge junction conditions [18].

In a series of recent papers [5]-[9], [16], [17], families of exact solutions have been considered which describe the propagation of gravitational waves in various backgrounds, together with the head-on collision of such waves and their subsequent interaction. These solutions may here be re-formulated using solutions of (6.4) in the form

$$
V(f, g)=V_{0}(f, g)+\Theta(\eta-\mu) \int_{1 / 2}^{\infty} \phi(k)(f+g)^{k} H_{k}\left(\frac{g-f}{f+g}\right) d k
$$

where $V_{0}(f, g)$ is a solution of (6.4) representing some particular background space-time, $\Theta(\eta-\mu)$ is the Heaviside step function in which the wavefront $f=0$ is assumed to be given by $\eta-\mu=0$, and $\phi(k)$ is an arbitrary function. The function $\phi(k)$ may be regarded as the "spectral amplitude" of some arbitrary wave profile. It is subject only to the condition that the above integral exists.

Let us now turn to the specific cases in which the backgrounds are the various FRW models with a stiff perfect fluid source. In these cases, the background regions of course are conformally flat. However, the wave regions are algebraically general indicating that the propagating gravitational wave is necessarily backscattered. 


\section{$7 \quad$ Plane waves in a spatially flat FRW model}

In terms of the metric (1.1), the spatially flat FRW universe with a stiff fluid is given by (5.4) and (5.11). Thus $e^{-U}=2 \eta$, and we can put

$$
f=\eta-z, \quad g=\eta+z .
$$

We may thus consider a plane wavefront given by $\eta=z$ as described in Section 2 , with the region $\eta<z$ being an exact FRW background into which a gravitational wave propagates.

Since $V=0$ in the background region in this case, the gravitational wave can be introduced by putting

$$
V(\eta, z)=\Theta(\eta-z) \int_{1 / 2}^{\infty} \phi(k) \eta^{k} H_{k}\left(\frac{z}{\eta}\right) d k
$$

in which a factor of $2^{k}$ has been absorbed into the arbitrary function $\phi(k)$, and $H_{k}(z / \eta)$ is given by (6.12) and its extension. Using the method described in [9] and [17, the complete integral for the metric function $M$ can be expressed as

$$
M(\eta, z)=-\log 2 \eta+\tilde{M} \Theta(\eta-z)
$$

where

$$
\tilde{M}=-\frac{1}{2} \int_{1}^{\infty} \frac{\eta^{n}}{n} d n \int_{1 / 2}^{n-1 / 2} \phi(k) \phi(n-k)\left[k(n-k) H_{k} H_{n-k}+\left(1-\frac{z^{2}}{\eta^{2}}\right) H_{k-1} H_{n-k-1}\right] d k,
$$

in which the argument of $H_{k}(z / \eta)$ has been omitted.

The non-zero components of the Weyl tensor in this case are given by

$$
\begin{aligned}
& \Psi_{0}=-\frac{1}{8 \eta} e^{\tilde{M}}\left[V_{\eta \eta}+2 V_{\eta z}+V_{z z}-\frac{\eta}{2}\left(V_{\eta}+V_{z}\right)^{3}\right], \\
& \Psi_{2}=\frac{1}{16 \eta} e^{\tilde{M}}\left[V_{\eta}^{2}-V_{z}^{2}\right], \\
& \Psi_{4}=-\frac{1}{8 \eta} e^{\tilde{M}}\left[V_{\eta \eta}-2 V_{\eta z}+V_{z z}-\frac{\eta}{2}\left(V_{\eta}-V_{z}\right)^{3}\right] .
\end{aligned}
$$

These are zero in the background region. Near the wavefront where $\eta-z$ is small, their behaviour is governed by the lowest non-zero term in $k$ in (7.2). Taking this lowest term in the form

$$
\begin{aligned}
V(\eta, z) & =\frac{a_{k}}{c_{k}} 2^{k} \eta^{k} H_{k}\left(\frac{z}{\eta}\right) \Theta(\eta-z) \\
& =a_{k} \frac{(\eta-z)^{k+1 / 2}}{(2 \eta)^{1 / 2}} F\left(\frac{1}{2}, \frac{1}{2} ; \frac{3}{2}+k ; \frac{1}{2}\left(1-\frac{z}{\eta}\right)\right) \Theta(\eta-z),
\end{aligned}
$$

where $a_{k}$ is a constant, it can be seen that the Weyl tensor components behave near the wavefront as

$$
\begin{aligned}
& \Psi_{4} \sim-\frac{a_{k}\left(k+\frac{1}{2}\right)}{(2 \eta)^{3 / 2}}(\eta-z)^{k-1 / 2} \delta(\eta-z)-\frac{a_{k}\left(k^{2}-\frac{1}{4}\right)}{(2 \eta)^{3 / 2}}(\eta-z)^{k-3 / 2} \Theta(\eta-z), \\
& \Psi_{2} \sim-\frac{a_{k}^{2}\left(k+\frac{1}{2}\right)}{32 \eta^{3}}(\eta-z)^{2 k} \Theta(\eta-z), \\
& \Psi_{0} \sim-\frac{3 a_{k}}{4(2 \eta)^{7 / 2}}(\eta-z)^{k+1 / 2} \Theta(\eta-z),
\end{aligned}
$$


where $\delta(\eta-z)$ is the Dirac delta function. This immediately identifies $\Psi_{4}$ as the component representing the propagating wave with wavefront $\eta=z, \Psi_{0}$ is the backscattered wave component propagating in the opposite spatial direction, while $\Psi_{2}$ represents the effective interaction between these two waves. It can also be seen that an impulsive gravitational wave is included if the lowest term in the expansion for $V$ has $k=\frac{1}{2}$. It has a step (or shock) gravitational wave if the lowest term has $k=\frac{3}{2}$. In general, the Weyl tensor is $C^{n}$ across the wavefront if $k_{\min }=n+\frac{5}{2}$.

It can be shown that this solution is regular everywhere except at the initial "big bang" singularity $\eta=0$, where both the waves and the background may be considered to originate.

These solutions were first considered in [5] for the case in which $k$ takes half integer values only. However, the complete solution was not obtained and the possible existence of an impulsive component was not recognised. The complete solution for a sum of terms with integer values of $k$ only was given in [9]. The head-on collision of such waves was discussed in [6] using half integer values of $k$ only, but an explicit expression for the function $M$ in the interaction region cannot be obtained by the methods considered so far.

\section{Cylindrical waves in a spatially flat FRW model}

The above method can also be used to construct cylindrical gravitational waves in a spatially flat FRW universe with a stiff fluid by expressing it using the coordinates defined in case $1 \mathrm{~b}$ of Section 5. In this case, the metric functions are given in the form (5.5) and the fluid by (5.11). Since $e^{-U}=2 \eta \rho$ in this case, we can put

$$
f=-\frac{1}{2}(\eta-\rho)^{2}, \quad g=\frac{1}{2}(\eta+\rho)^{2} .
$$

We may now consider a gravitational wave with a cylindrical wavefront given by $\eta=\rho$ as described in Section 2. Accordingly, the region $\eta<\rho$ is taken to be the exact FRW background into which the wave propagates. Such a wave may be considered to originate on a line $\rho=0$ at the initial singularity $\eta=0$. In this case $V=\log \rho$ in the background region, and a gravitational wave in the general form (6.14) can be introduced by putting

$$
V(\eta, \rho)=\log \rho+\Theta(\eta-\rho) \int_{1 / 2}^{\infty} \phi(k) \eta^{k} \rho^{k} H_{k}\left(\frac{\eta^{2}+\rho^{2}}{2 \eta \rho}\right) d k
$$

in which a factor of $2^{k}$ has again been absorbed into the arbitrary function $\phi(k)$. (This function, representing a spectral amplitude, should not be confused with the cylindrical coordinate being used in this case.) The functions $H_{k}\left(\frac{\eta^{2}+\rho^{2}}{2 \eta \rho}\right)$ are most conveniently given here by (6.11). Putting

$$
V=\log \rho+\tilde{V} \Theta(\eta-\rho), \quad M=-\log 2 \eta+\tilde{M} \Theta(\eta-\rho),
$$

it can be shown that the subsidiary equations (6.7) can be fully integrated to yield

$$
\tilde{M}=-\frac{1}{2} \int_{1 / 2}^{\infty} \phi(k) \eta^{k} \rho^{k}\left[H_{k}+\frac{\left(\eta^{2}-\rho^{2}\right)}{2 \eta \rho} \frac{1}{k} H_{k-1}\right] d k
$$




$$
\begin{aligned}
-\frac{1}{2} \int_{1}^{\infty} \frac{\eta^{n} \rho^{n}}{n} d n \int_{1 / 2}^{n-1 / 2} \phi(k) \phi(n-k) & \\
& \quad\left[k(n-k) H_{k} H_{n-k}-\frac{\left(\eta^{2}-\rho^{2}\right)^{2}}{4 \eta^{2} \rho^{2}} H_{k-1} H_{n-k-1}\right] d k,
\end{aligned}
$$

in which the argument of $H_{k}$ is taken to be $\frac{\eta^{2}+\rho^{2}}{2 \eta \rho}$.

In this case, the non-zero components of the Weyl tensor in the region $\eta \geq \rho$ are given by

$$
\begin{aligned}
& \Psi_{0}=-\frac{1}{8 \eta} e^{\tilde{M}}\left[\tilde{V}_{\eta \eta}+2 \tilde{V}_{\eta \rho}+\tilde{V}_{\rho \rho}+\frac{1}{\eta+\rho}\left(\tilde{V}_{\eta}+\tilde{V}_{\rho}\right)-\frac{3 \eta}{2(\eta+\rho)}\left(\tilde{V}_{\eta}+\tilde{V}_{\rho}\right)^{2}-\frac{\eta \rho}{2(\eta+\rho)}\left(\tilde{V}_{\eta}+\tilde{V}_{\rho}\right)^{3}\right], \\
& \Psi_{2}=-\frac{1}{16 \eta} e^{\tilde{M}}\left[\tilde{V}_{\eta}^{2}-\tilde{V}_{\rho}^{2}-\frac{2}{\rho} \tilde{V}_{\rho}\right], \\
& \Psi_{4}=-\frac{1}{8 \eta} e^{\tilde{M}}\left[\tilde{V}_{\eta \eta}-2 \tilde{V}_{\eta \rho}+\tilde{V}_{\rho \rho}+\frac{1}{\eta-\rho}\left(\tilde{V}_{\eta}-\tilde{V}_{\rho}\right)-\frac{3 \eta}{2(\eta-\rho)}\left(\tilde{V}_{\eta}-\tilde{V}_{\rho}\right)^{2}+\frac{\eta \rho}{2(\eta-\rho)}\left(\tilde{V}_{\eta}-\tilde{V}_{\rho}\right)^{3}\right] .
\end{aligned}
$$

The term $-\frac{1}{8 \eta} e^{\tilde{M}}\left(\tilde{V}_{\eta}-\tilde{V}_{\rho}\right) \delta(\eta-\rho)$ which would normally appear in the expression for $\Psi_{4}$ has been omitted because, in this case, $\tilde{V}_{\eta}=\tilde{V}_{\rho}$ on the wavefront $\eta=\rho$. It follows that this class of solutions does not include impulsive cylindrical waves.

Near the wavefront where $\eta-\rho$ is small, the character of the gravitational wave is determined by the lowest non-zero term in $k$ in (8.2). Taking this lowest term in the form

$$
\begin{aligned}
\tilde{V}(\eta, z) & =\frac{a_{k}}{c_{k}} 2^{2 k} \eta^{k} \rho^{k} H_{k}\left(\frac{\eta^{2}+\rho^{2}}{2 \eta \rho}\right) \\
& =a_{k} \frac{(\eta-\rho)^{1+2 k}}{(\eta+\rho)} F\left(\frac{1}{2}, 1+k ; \frac{3}{2}+k ;\left(\frac{\eta-\rho}{\eta+\rho}\right)^{2}\right),
\end{aligned}
$$

it can be seen that, near the wavefront, the Weyl tensor components behave as

$$
\begin{aligned}
\Psi_{4} & \sim-\frac{1}{8}(2 k+1)(4 k+1) a_{k} \eta^{-2}(\eta-\rho)^{2 k-1} \Theta(\eta-\rho), \\
\Psi_{2} & \sim-\frac{1}{16}(2 k+1) a_{k} \eta^{-3}(\eta-\rho)^{2 k} \Theta(\eta-\rho), \\
\Psi_{0} & \sim-\frac{3}{32} a_{k} \eta^{-4}(\eta-\rho)^{2 k+1} \Theta(\eta-\rho) .
\end{aligned}
$$

It can thus be seen that these solutions include a step (or shock) gravitational wave if the lowest term in the expansion for $V$ has $k=\frac{1}{2}$. Generally, the Weyl tensor is $C^{n}$ across the wavefront if $k_{\min }=\frac{1}{2} n+1$.

It can be shown that $\tilde{V}$ and $\tilde{M}$ and their derivatives with respect to $\eta$ and $\rho$ are all bounded as $\rho \rightarrow 0$ for all $\eta>0$. It thus follows from (8.5) that the component $\Psi_{2}$ of the Weyl tensor is singular on the axis $\rho=0$. Thus the axis of the cylindrical waves is a scalar curvature singularity even though the fluid density on it given by (5.11) is bounded. This axis may be considered both as the source of the cylindrical waves, and also as a "sink" of the backscattered radiation.

The possibility of obtaining exact solutions of this type was mentioned in [9] but the explicit solutions are given here for the first time. By superposing two sets of terms similar to those included in (8.2) but with wavefronts given by $\eta=\rho-a$ and $\eta=b-\rho$ for positive constants $a$ and $b$, it is also possible to consider the collision of outgoing and incoming cylindrical gravitational waves. This is qualitatively described in figure 3 . Exact solutions describing this situation are only singular on an initial hypersurface and on the axis of symmetry. However, although the qualitative features of such a solution can be determined, the complete integral for $M$ cannot be obtained in this case using the methods described above. 


\section{Plane waves in an open FRW model}

We now turn to the case in which a plane gravitational wave propagates in an open FRW universe with a stiff perfect fluid. In this case, it is convenient to adopt the coordinates $\eta, \mu, x, y$ as defined in case $2 \mathrm{a}$ of Section 5 . In the background region, the metric functions are given by (5.6) and the fluid by (5.12). Since $e^{-U}=\sinh 2 \eta e^{-2 \mu}$ in this case, we can put

$$
f=\frac{1}{2}\left(e^{2(\eta-\mu)}-1\right), \quad g=\frac{1}{2}\left(1-e^{-2(\eta+\mu)}\right) .
$$

We can now consider a gravitational wave with a plane wavefront given by $\eta=\mu$ as described in Section 3, with the region $\eta<\mu$ being part of the exact open FRW model into which it propagates.

Since $V=0$ in the background region in this case, the gravitational wave can be introduced by putting

$$
V(\eta, \mu)=\Theta(\eta-\mu) \int_{1 / 2}^{\infty} \phi(k) \sinh ^{k} 2 \eta e^{-2 k \mu} H_{k}\left(\frac{e^{2 \mu}-\cosh 2 \eta}{\sinh 2 \eta}\right) d k,
$$

where $H_{k}$ is most conveniently given by (6.12) and its extension. After applying again the method described in [9], the complete integral for $M$ is found to be

$$
M(\eta, \mu)=-\log \sinh 2 \eta+\tilde{M} \Theta(\eta-\mu)
$$

where

$$
\begin{aligned}
\tilde{M}=-\frac{1}{2} \int_{1}^{\infty} \sinh ^{n} 2 \eta e^{-2 n \mu} \frac{1}{n} d n \int_{1 / 2}^{n-1 / 2} \phi(k) \phi(n-k) \\
\\
{\left[k(n-k) H_{k} H_{n-k}-\left(\frac{1-2 \cosh 2 \eta e^{2 \mu}+e^{4 \mu}}{\sinh ^{2} 2 \eta}\right) H_{k-1} H_{n-k-1}\right] d k, }
\end{aligned}
$$

in which the argument of $H_{k}$ is $\left(e^{2 \mu}-\cosh 2 \eta\right) / \sinh 2 \eta$.

As previously, we can determine the behaviour of the gravitational wave near the wavefront where $\eta-\mu$ is small by considering the lowest non-zero term in $k$ in (9.2). Taking this lowest term in the form

$$
V(\eta, \mu)=\frac{a_{k}}{c_{k}} \sinh ^{k} 2 \eta e^{-2 k \mu} H_{k}\left(\frac{e^{2 \mu}-\cosh 2 \eta}{\sinh 2 \eta}\right) \Theta(\eta-z),
$$

it can be seen that, near the wavefront, the non-zero Weyl tensor components behave as

$$
\begin{aligned}
& \Psi_{4} \sim-\frac{a_{k}\left(k+\frac{1}{2}\right)}{2^{1 / 2}\left(e^{4 \eta}-1\right)^{3 / 2}}(\eta-\mu)^{k-1 / 2} \delta(\eta-\mu)-\frac{a_{k}\left(k^{2}-\frac{1}{4}\right)}{2^{1 / 2}\left(e^{4 \eta}-1\right)^{3 / 2}}(\eta-\mu)^{k-3 / 2} \Theta(\eta-\mu), \\
& \Psi_{2} \sim-\frac{a_{k}^{2}\left(k+\frac{1}{2}\right)}{4 \sinh ^{3} 2 \eta}(\eta-\mu)^{2 k} \Theta(\eta-\mu), \\
& \Psi_{0} \sim-\frac{3 a_{k} e^{5 \eta}}{2 \sinh ^{7 / 2} 2 \eta}(\eta-\mu)^{k+1 / 2} \Theta(\eta-\mu) .
\end{aligned}
$$

It can thus be seen that an impulsive gravitational wave is again included here if the lowest term in the expansion for $V$ has $k=\frac{1}{2}$. It has a step (or shock) gravitational wave if the lowest term has $k=\frac{3}{2}$. Generally, the Weyl tensor is $C^{n}$ across the wavefront if $k_{\min }=n+\frac{5}{2}$. 
From the above results, it can be seen that these waves in open FRW universes are qualitatively similar to those in spatially flat FRW universes. They both include impulsive, shock or smooth fronted wavefronts. As discussed in Section 3, the geometrical properties of these plane wavefronts in open FRW universes are described in figure 1 and equation (3.7).

A discussion of these waves and their wavefonts, as well as the possible collision of such waves, was given in [7]. However, the complete solution for $M$ and an analysis of the character of the Weyl tensor components near the wavefront has been included here for the first time.

\section{Cylindrical waves in an open FRW model}

We now consider a gravitational wave which propagates into a stiff fluid open FRW universe along the null cylindrical wavefronts described in figure 2 and Eq. (3.10). In this case, it is convenient to adopt the coordinates $\eta, \rho, \phi, z$ as defined in case $2 \mathrm{~b}$ of Section 5 . In the background region, the metric functions are given by (5.7) and the fluid by (5.12). Since $e^{-U}=\sinh 2 \eta \sinh 2 \rho$ in this case, we can put

$$
f=-\sinh ^{2}(\eta-\rho), \quad g=\sinh ^{2}(\eta+\rho) .
$$

We can now consider a gravitational wave with a cylindrical wavefront $\eta=\rho$ as described in Section 3, with the region $\eta<\rho$ being part of the exact open FRW universe into which the wave propagates. This can be achieved by putting

$$
V(\eta, \rho)=\log \tanh \rho+\tilde{V}(\eta, \rho) \Theta(\eta-\rho)
$$

where

$$
\tilde{V}(\eta, \rho)=\int_{1 / 2}^{\infty} \phi(k) \sinh ^{k} 2 \eta \sinh ^{k} 2 \rho H_{k}\left(\frac{\cosh 2 \eta \cosh 2 \rho-1}{\sinh 2 \eta \sinh 2 \rho}\right) d k,
$$

in which $H_{k}$ is now most conveniently given by (6.11). In this case the integration of the

subsidiary equations (6.7) is more complicated. However, the complete integral for $M$ can still be obtained and expressed in the form

$$
M(\eta, \rho)=-\log \sinh 2 \eta+\tilde{M}(\eta, \rho) \Theta(\eta-\rho),
$$

where

$$
\begin{gathered}
\tilde{M}=-\frac{1}{2} \int_{1 / 2}^{\infty} \phi(k)\left\{2 k A_{k-1}(\tau, \zeta) H_{k}+\left[\left(\zeta^{2}-1\right)^{1 / 2} \frac{1}{k} \tau^{k}+\left(\zeta^{2}-1\right) A_{k}(\tau, \zeta)\right] H_{k-1}\right\} d k \\
-\frac{1}{2} \int_{1}^{\infty} \frac{1}{n} \tau^{n} d n \int_{1 / 2}^{n-1 / 2} \phi(k) \phi(n-k) \\
\quad\left[k(n-k) H_{k} H_{n-k}-\left(\zeta^{2}-1\right) H_{k-1} H_{n-k-1}\right] d k
\end{gathered}
$$

in which

$$
A_{k}(\tau, \zeta)=\int_{0}^{\tau} \frac{t^{k}}{\left[(2+\zeta t)^{2}-t^{2}\right]^{1 / 2}} d t
$$


$H_{k}=H_{k}(\zeta)$ are as defined above, and $\tau$ and $\zeta$ are functions of $\eta$ and $\rho$ given by

$$
\tau=\sinh 2 \eta \sinh 2 \rho, \quad \zeta=\frac{\cosh 2 \eta \cosh 2 \rho-1}{\sinh 2 \eta \sinh 2 \rho} .
$$

It can be seen that these solutions are singular at the initial big bang at which $\eta=0$, and that there is an additional curvature singularity on the axis of symmetry $\rho=0$. The singular axis can be considered to represent both the source of the cylindrical gravitational wave and the sink of the backscattered radiation, as in the spatially flat case described in Section 8 .

It is also possible to analyse the character of the wave along the wavefront using the same method as in previous sections. Accordingly, we include only the lowest non-zero term in $k$ in (10.2) in the form

$$
\tilde{V}(\eta, \rho)=\frac{a_{k}}{c_{k}} \sinh ^{k} 2 \eta \sinh ^{k} 2 \rho H_{k}\left(\frac{\cosh 2 \eta \cosh 2 \rho-1}{\sinh 2 \eta \sinh 2 \rho}\right) .
$$

With this it can be shown that, near the wavefront, the non-zero Weyl tensor components behave as

$$
\begin{aligned}
& \Psi_{4} \sim-\frac{a_{k}(2 k+1)(4 k+1)}{2 \sinh ^{2} 2 \eta}(\eta-\rho)^{2 k-1} \Theta(\eta-\rho), \\
& \Psi_{2} \sim \frac{a_{k}(2 k+1)}{2 \sinh ^{3} 2 \eta}(\eta-\rho)^{2 k} \Theta(\eta-\rho), \\
& \Psi_{0} \sim-\frac{3 a_{k}}{2 \sinh ^{4} 2 \eta}(\eta-\rho)^{2 k+1} \Theta(\eta-\rho) .
\end{aligned}
$$

These expressions can be seen to be very similar to those for cylindrical waves in the spatially flat FRW universe as described in Section 8 (see (8.7) in particular). As there, it can be seen that these solutions include a step (or shock) gravitational wave if the lowest term in the expansion for $V$ has $k=\frac{1}{2}$. Generally, the Weyl tensor is $C^{n}$ across the wavefront if $k_{\min }=\frac{1}{2} n+1$.

The possibility of obtaining explicit solutions of this type was mentioned in [9] but the solutions are described in detail here for the first time. It is also possible to consider the collision of opposing outgoing and incoming cylindrical gravitational waves just as in the spatially flat case. However, although the qualitative features of such a situation can be determined, a complete integral for $M$ in the interaction region cannot be obtained in this case using the above method.

\section{Toroidal waves in a closed FRW model}

We now consider waves in a closed FRW universe. In this case, no plane-fronted waves can exist. However, as described in Section 4, waves with toroidal surfaces can be constructed. These are analogous with the cylindrical-fronted waves in an open universe considered in the previous section. Mathematically, they can be obtained by replacing the hyperbolic functions by the corresponding trigonometric functions. Physically, the cylindrical wavefront becomes closed to form a toroidal wavefront. 
In this case, it is convenient to adopt the coordinates $\eta, \zeta, \delta, \sigma$ as defined in case 3 of Section 5. Starting from the background (5.8), we may consider the family of null hypersurfaces $\eta-\zeta=$ const. At any fixed time $\eta=$ const., the wave surface is a toroid $\zeta=$ const. However, as explained in Section 4 , two axes now exist - at $\zeta=0$ and at $\zeta=\pi / 2$. We may consider a wave starting at the initial big bang singularity $(\eta=0)$ at the circular axis $\zeta=0$, and propagating along the null hypersurface $\eta=\zeta$ into the FRW closed universe. At any fixed time $\eta$, the wavefront will be a toroidal 2 -surface as illustrated in figure 4. As the universe expands, the front of the wave propagates towards the (circular) axis $\zeta=\pi / 2$.

In the background region, the metric functions are given by (5.8) and the fluid by (5.13). Since $e^{-U}=\sin 2 \eta \sin 2 \zeta$ in this case, we can put

$$
f=-\sin ^{2}(\eta-\zeta), \quad g=\sin ^{2}(\eta+\zeta) .
$$

The gravitational wave with the toroidal wavefront $\eta=\zeta$ is determined by putting

$$
V(\eta, \zeta)=\log \tan \zeta+\tilde{V}(\eta, \zeta) \Theta(\eta-\zeta)
$$

where

$$
\tilde{V}(\eta, \zeta)=\int_{1 / 2}^{\infty} \phi(k) \sin ^{k} 2 \eta \sin ^{k} 2 \zeta H_{k}\left(\frac{1-\cos 2 \eta \cos 2 \zeta}{\sin 2 \eta \sin 2 \zeta}\right) d k
$$

in which $H_{k}$ is given by (6.11). As above, it can be shown that the complete integral for $M$ can be expressed in the form

$$
M(\eta, \zeta)=-\log \sin 2 \eta+\tilde{M}(\eta, \zeta) \Theta(\eta-\zeta)
$$

where

$$
\begin{gathered}
\tilde{M}=-\frac{1}{2} \int_{1 / 2}^{\infty} \phi(k)\left\{2 k B_{k-1}(\tau, \xi) H_{k}+\left[\left(\xi^{2}-1\right)^{1 / 2} \frac{1}{k} \tau^{k}-\left(\xi^{2}-1\right) B_{k}(\tau, \xi)\right] H_{k-1}\right\} d k \\
-\frac{1}{2} \int_{1}^{\infty} \frac{1}{n} \tau^{n} d n \int_{1 / 2}^{n-1 / 2} \phi(k) \phi(n-k) \\
\quad\left[k(n-k) H_{k} H_{n-k}-\left(\xi^{2}-1\right) H_{k-1} H_{n-k-1}\right] d k
\end{gathered}
$$

in which

$$
B_{k}(\tau, \xi)=\int_{0}^{\tau} \frac{t^{k}}{\left[(2-\xi t)^{2}-t^{2}\right]^{1 / 2}} d t
$$

and $\tau$ and $\xi$ are functions of $\eta$ and $\zeta$ given by

$$
\tau=\sin 2 \eta \sin 2 \zeta, \quad \xi=\frac{1-\cos 2 \eta \cos 2 \zeta}{\sin 2 \eta \sin 2 \zeta},
$$

and to avoid confusion with the coordinate $\zeta$, in this case, we have relabelled the argument of $H_{k}$ putting $H_{k}=H_{k}(\xi)$.

The character of the wave along the wavefront can also be analysed as in previous sections. Accordingly, we consider the lowest non-zero term in $k$ in (11.2) in the form

$$
\tilde{V}(\eta, \zeta)=\frac{a_{k}}{c_{k}} \sin ^{k} 2 \eta \sin ^{k} 2 \zeta H_{k}\left(\frac{1-\cos 2 \eta \cos 2 \zeta}{\sin 2 \eta \sin 2 \zeta}\right) .
$$


With this it can be shown that, near the wavefront, the non-zero Weyl tensor components behave as

$$
\begin{aligned}
& \Psi_{4} \sim-\frac{a_{k}(2 k+1)(4 k+1)}{2 \sin ^{2} 2 \eta}(\eta-\zeta)^{2 k-1} \Theta(\eta-\zeta), \\
& \Psi_{2} \sim \frac{a_{k}(2 k+1)}{2 \sin ^{3} 2 \eta}(\eta-\zeta)^{2 k} \Theta(\eta-\zeta), \\
& \Psi_{0} \sim-\frac{3 a_{k}}{2 \sin ^{4} 2 \eta}(\eta-\zeta)^{2 k+1} \Theta(\eta-\zeta) .
\end{aligned}
$$

These expressions are obvious modifications of those for cylindrical waves in spatially flat and open FRW universes as given by (8.7) and (10.9). They include a step (or shock) gravitational wave if the lowest term in the expansion for $V$ has $k=\frac{1}{2}$. Generally, the Weyl tensor is $C^{n}$ across the wavefront if $k_{\min }=\frac{1}{2} n+1$.

It can be seen that these solutions are singular at the initial big bang at which $\eta=0$, and at the final big crunch at which $\eta=\pi / 2$. There is also an additional curvature singularity on the axis of symmetry $\zeta=0$, which can be considered to represent both the source of the toroidal gravitational wave and the sink of the backscattered radiation.

As noticed in Section 5, from the big bang until the big crunch, a photon in a closed universe with a stiff fluid succeeds in travelling only one quarter of the distance around the whole universe. Similarly, an exact shock wave starting at the big bang on the "polar axis" $\zeta=0$ will reconverge onto the "equatorial" axis $\zeta=\pi / 2$ at the big crunch. The space-time diagram of such a situation is given in figure 5 .

It is also possible to consider the collision of two shock waves propagating into the closed FRW background with a stiff fluid. At the big bang, one wave starts at the axis $\zeta=0$ and the other at $\zeta=\pi / 2$. As the universe expands, the waves approach each other and collide just at the time of maximum expansion. One may also consider other initial conditions with the waves being some distance from the axes at time $\eta=0$. Such waves will collide while the universe is still expanding. However, although the qualitative features of such a situation can be determined a complete integral for $M$ cannot be obtained in this case. Locally, such waves were considered in [8]. (The coordinates $z, x, y$ used in [8] are related to those above by $\zeta=z, \delta=2^{1 / 2} x, \sigma=2^{1 / 2} y$.) The toroidal character of the waves was, however, not noticed and the discussion of the singularities was incorrect.

Solutions of this type are closed universes with two global, commuting and hypersurface orthogonal Killing vectors. They may therefore be considered as Gowdy-type space-times [20], [21]. It can readily be verified that here the regularity conditions across the null hypersurface $\eta=\zeta$ are satisfied. Such conditions were studied carefully by Gowdy himself in the vacuum case, and by Kitchingham [22] (see also references therein) in the case of stiff fluid cosmologies.

\section{Concluding remarks}

Exact gravitational waves with two isometries have been constructed which can be considered as exact perturbations of FRW cosmological models with a stiff fluid. The waves have distinct wavefronts and propagate into any of the three possible FRW backgrounds. 
Although much work has been done on considering various types of waves in the context of cosmological models (c.f. reviews [1]-[何), waves with distinct wavefronts propagating into the standard FRW backgrounds have only recently been considered ([5]-[9]).

In the spatially flat and open universes, the wavefronts can be either plane or cylindrical. In the closed models, they are toroidal. One may consider toroidal waves propagating in other models as well, provided that the spatial sections are compactified by making appropriate identifications. However, such identifications result in anisotropic background spaces. Here we have assumed the standard backgrounds with natural topologies admitting globally the six-parameter group of isometries.

We have obtained exact solutions for plane-fronted gravitational waves propagating in a spatially flat or open FRW universe with a stiff fluid. These include impulsive waves, shock waves, or waves with Weyl tensor components of any arbitrary finite degree of smoothness. We have also presented exact solutions for cylindrical- or toroidal-fronted gravitational waves propagating in a spatially flat, open or closed FRW stiff fluid universe. These do not include impulsive waves, but may include shock waves or waves of any arbitrary finite degree of smoothness.

Solitonic perturbations of FRW models with cylindrical symmetry, without wavefronts, have commonly been treated in the literature (see e.g. [14] and [22], and references therein). The possibility of constructing plane-fronted waves in the open FRW universes appears to have been recognised only recently [0]. On the other hand, it is well known that the $G_{2}$ cosmological models, that are often interpreted as inhomogeneous generalisations of the FRW models, can sometimes also be used to describe cylindrical perturbations simply by reinterpreting the relevant coordinates. It can be seen from the above examples that, not only is the topological character of the wavefronts changed by this procedure, but the character of the wave may be altered as well.

No singularities, except those representing the big bang or big crunch, arise in the closed vacuum models considered by Gowdy [20, [21] or in the soliton-type waves studied by Belinskii [14], Kitchingham [22] and others (see [1], [3], [4] for references) in cases of both open and closed FRW stiff fluid models. Their explicit solutions, although without wavefronts, clearly correspond to our cylindrical waves, or to toroidal waves in the closed case. However, in the spatially flat and open FRW models, they could also be adapted to infinite plane perturbations.

With a clear geometrical picture of the wavefronts available, it may be of interest to consider also other types of shock waves with two isometries propagating into these FRW backgrounds. These may be just test radiative fields (not necessarily gravitational), or exact (large) perturbations. For example, one could discuss fluid shocks in all types of FRW models, as Tabensky and Taub [19] considered shock acoustic waves in the spatially flat universe in their pioneering work.

The advantage of studying shock gravitational waves with distinct wavefronts is not only in having a clear picture of the wave surfaces. One can also discuss the head-on collision of such waves. In the case of the spatially flat and open universes, the collision of waves with plane surfaces was analysed in detail in [6] and [7]. The presence of the gravitational waves slows down the rate of expansion, but future spacelike singularities do 
not occur for interacting waves in these expanding universes as they do in the vacuum case with a Minkowski background. This result is qualitatively similar to those of Centrella 23] and Centrella and Matzner [24] which study the collision of waves in an expanding vacuum Kasner background.

Similarly, one can consider the collision of "outgoing" and "incoming" waves with cylindrical wavefronts in the spatially flat and open universes, and toroidal wavefronts in the closed models. No future spacelike singularities seem to arise again. However, it appears that making the wave surfaces finite along one direction in the cylindrical case and fully finite in the toroidal case gives rise to timelike singularities representing the histories of the corresponding axes. The "source" at the axis appears to be needed to support the propagation of such waves.

\section{Appendix}

We here consider the geometry of possible wave surfaces in a closed FRW universe in greater detail. We will show that a typical wave surface is a 2-torus in the 3 -sphere described by (4.3) and (4.4). First consider a circle $S$, as illustrated in figure 6 , in the $W, Z$-plane given by

$$
S: \quad W^{2}+Z^{2}=\kappa^{2} R_{0}^{2}, \quad X=Y=0,
$$

where $\kappa \in[0,1]$ is a constant. The circle $S$ is generally inside the 3 -sphere. Next, consider a 2-plane given by

$$
(X, Y) \in \mathbb{R}^{2}, \quad W, Z \text { fixed, } \quad W^{2}+Z^{2}=\kappa^{2} R_{0}^{2}
$$

Such a plane for any fixed $W$ and $Z$ touches the circle $S$ at just one point, and it intersects the 3 -sphere in the circle

$$
X^{2}+Y^{2}=\left(1-\kappa^{2}\right) R_{0}^{2},
$$

with $W, Z$ fixed and satisfying $W^{2}+Z^{2}=\kappa^{2} R_{0}^{2}$. When $W=\kappa R_{0}$ and $Z=0$, this is the circle $C$ illustrated in figure 7 . In figure 6 only two points $a$ and $b$ of this circle can be seen because one dimension has been suppressed. Now by moving the point $(W, X)$ along the circle $S$ and considering the corresponding family of 2-planes (A.2), we find that the planes intersect the 3 -sphere in the 2 -torus $T^{2}=S^{1} \otimes S^{1}$ given by

$$
W^{2}+Z^{2}=\kappa^{2} R_{0}^{2}, \quad X^{2}+Y^{2}=\left(1-\kappa^{2}\right) R_{0}^{2} .
$$

In figure 6 , the slice $Y=0$ through the 2-torus is seen as two circles, $A$ and $B$, given by (A.4) with $Y=0$. These circles represent the intersection of the 2 -sphere $W^{2}+$ $Z^{2}+X^{2}=R_{0}^{2}$ through the 2-torus (A.4). In a universe with large $R_{0}$, the circles will locally approximate to straight lines and, if $\kappa$ is close to 1 , the surfaces will appear to be cylindrical.

Suppressing the coordinate $Z$, the 2 -torus is represented by the circles $X^{2}+Y^{2}=$ $\left(1-\kappa^{2}\right) R_{0}^{2}, W= \pm \kappa R_{0}$, denoted by $C$ and $D$ in figure 7 . These two circles represent the intersection of the 2-sphere $X^{2}+Y^{2}+W^{2}=R_{0}^{2}$ through the 2-torus (A.4). 
It is seen from (A.4) that the 2-torus is specified by the choice of the parameter $\kappa$ which may conveniently be written as $\kappa=\cos \zeta$, with $0 \leq \zeta \leq \pi / 2$. With the form of (A.4), the natural coordinates on the 2-torus are the angles, say $\sigma$ and $\delta$, parametrizing the two circles of radii $R_{0} \cos \zeta$ and $R_{0} \sin \zeta$. This leads to the alternative parametrization of the whole 3 -sphere given by

$$
\begin{aligned}
W & =R_{0} \cos \zeta \cos \sigma \\
Z & =R_{0} \cos \zeta \sin \sigma \\
X & =R_{0} \sin \zeta \cos \delta \\
Y & =R_{0} \sin \zeta \sin \delta
\end{aligned}
$$

where $\zeta \in[0, \pi / 2], \sigma \in[0,2 \pi], \delta \in[0,2 \pi]$. In figure 6 , the two families of circles $A$ and $B$, each of radius $R_{0} \cos \zeta$, have $\delta=0$ and $\delta=\pi$ respectively, and are parametrized by $0 \leq \sigma \leq 2 \pi$. Similarly, the families of circles $C$ and $D$, illustrated in figure 7 , have radius $R_{0} \sin \zeta$, with $\sigma=0$ and $\sigma=\pi$ respectively, and are parametrized by $0 \leq \delta \leq 2 \pi$. Thus, this is a natural parametrization for the description of toroidal 2-surfaces inside the 3 sphere. Notice that degenerate points occur in both figures either when $\zeta=0$, or when $\zeta=\pi / 2$. These are coordinate singularities in which the norms of the Killing vectors $\partial_{\delta}$ or $\partial_{\sigma}$ vanish. It is easily verified that the 3 -sphere is covered once with the angular variables $\zeta, \sigma, \delta$ taking values in the given intervals. On the 3 -sphere, these angular variables are related to the familiar Euler angular coordinates $\theta, \phi, \psi$ (which should not be confused with the angles used in (4.1) etc.) by $\theta=2 \zeta, \phi=\sigma-\delta, \psi=\sigma+\delta$. The Euler angles are also often used since the 3 -sphere has the same topology as the group $S U(2)$, and the generators of the symmetries can be suitably expressed in terms of these angles (see e.g. 20] or 25]).

It is possible to consider the 2-torus (A.4) as dividing the 3 -sphere into two solid tori. Conversely, $S^{3}$ can be constructed by identifying the boundaries of two solid tori. This appears to have been first explained in relativity literature by Misner [26] in his analysis of Taub-NUT space, using an inversion. We will now demonstrate this, using the parametrization (A.1), thus making apparent the character of the coordinates $\zeta, \sigma$ and $\delta$. (See also Appendix B of [20].)

We first cut the 3-sphere (4.3) into two along the hypersurface $Z=0$, or $\sigma=0$ and $\sigma=\pi$, to form two solid 2-spheres. (This is similar to cutting an ordinary 2-sphere along an equatorial plane to form two hemispheres which can each be mapped, by stereographic projection, onto a disc.) This is illustrated in figure 8 , in which the solid 2-sphere on the left corresponds to the part of the 3 -sphere in which $Z \geq 0$, that on the right to $Z \leq 0$. The two spheres are reconnected by identifying corresponding points on their surfaces.

The full curves inside the two spheres indicated in figure 8 represent the section of the sphere in which $\delta=0$ and $\delta=\pi$ for some fixed value of $\zeta$. They can be seen to arise from the stereographic projection of the hemispheres with $Z \geq 0$ and $Z \leq 0$ in figure 6 onto the $(W, X)$ plane, the centre of projection being the points $W=X=0, Z=-R_{0}$ and $W=X=0, Z=+R_{0}$. Introducing coordinates $\tilde{W}, \tilde{X}$ for the projected points of the hemisphere with, say $Z \geq 0, \tilde{W}=W R_{0} /\left(R_{0}+Z\right), \tilde{X}=X R_{0} /\left(R_{0}+Z\right)$, and substituting for $W, X$ and $Z$ from (A.5) with $\delta=0$, we obtain $\tilde{W}^{2}+\left(\tilde{X}-R_{0} / \sin \zeta\right)^{2}=R_{0}^{2} \cot \zeta$. 
For fixed values of $\zeta$, these are segments of circles, such as $A$ in figure 8 , with centres on $\tilde{X}>0$, bounded by $Z=0$. Along these segments $\sigma$ is changing and $\delta=0$. As $\zeta \rightarrow \pi / 2$, the segments degenerate to the "East pole" on the equator. It can thus be seen that the circles $A$ and $B$ in figure 6 are projected into the corresponding circles in figure 8.

The surfaces of both solid 2-spheres, defined by $Z=0$, must be identified in order to reconstruct the original 3-sphere. In figure 8, such an identification is illustrated in terms of the coordinates $\zeta, \sigma$ and $\delta$. Surfaces inside each sphere on which $\zeta$ is constant, as indicated in figure 8 , are joined on the surface of each sphere, dividing the 3 -sphere into two solid tori. One torus has the closed axis given by $\zeta=0$ and is formed by the axes of the two spheres illustrated in figure 8. On this axis $\sigma$ changes from 0 to $\pi$ in the left sphere and continues from $\pi$ up to $2 \pi$ in the right sphere. Along this axis, the coordinate $\delta$ is degenerate and the norm of the Killing vector $\partial_{\delta}$ vanishes. The second torus is evident on identifying points near the equators of both spheres. The equators form the second closed axis. It is given by $\zeta=\pi / 2$ and is parametrized by $0 \leq \delta \leq 2 \pi$, while the coordinate $\sigma$ is degenerate and the norm of the Killing vector $\partial_{\sigma}$ vanishes. These two axes, $\zeta=0$ and $\zeta=\pi / 2$, are crucial in dealing with toroidal waves in closed FRW universes.

\section{acknowledgements}

JB was supported by an award from the Royal Society and, in part, by the grant GACR202/96/0206 of the Czech Republic and the U.S.-Czech Science and Technology grant no. 92067.

\section{References}

[1] M. Carmeli, Ch. Charach and S. Malin, Phys. Rep. 76 (1981), 79.

[2] P. J. Adams, R. W. Hellings, R. L. Zimmerman, H. Farhoosh, D. I. Levine, and S. Zeldich, Astrophys. J. 253 (1982), 1.

[3] E. Verdaguer, Phys. Rep. 229 (1993), 1.

[4] A. Krasiński, "Inhomogeneous cosmological models", Cambridge University Press, Cambridge, 1996.

[5] J. B. Griffiths, Class. Quantum Grav. 10 (1993), 975.

[6] J. B. Griffiths, J. Math. Phys. 34 (1993), 4064.

[7] J. Bičák and J. B. Griffiths, Phys. Rev. D 49 (1994), 900.

[8] A. Feinstein and J. B. Griffiths, Class. Quantum Grav. 11 (1994), L109.

[9] G. A. Alekseev and J. B. Griffiths, Phys. Rev. D 52 (1995), 4497.

[10] C. Misner, K. S. Thorne and J. A. Wheeler, "Gravitation", Freeman, San Francisco, 1973. 
[11] Ya. B. Zeldovich, Sov. Phys. JETP 14 (1962), 1143.

[12] J. D. Barrow, Nature 272 (1978), 211.

[13] J. D. Walecka, Ann. Phys. (N.Y.) 83 (1974), 491.

[14] V. A. Belinskii, Zh. Eksp. Teor. Fiz. 77 (1979), 1239; Sov. Phys. JETP 50 (1979), 623 .

[15] J. Wainwright, W. C. W. Ince and B. J. Marshman, Gen. Rel. Grav. 10 (1979), 259.

[16] G. A. Alekseev and J. B. Griffiths, Class. Quantum Grav. 13 (1996), L13.

[17] G. A. Alekseev and J. B. Griffiths, Class. Quantum Grav. 13 (1996), to appear.

[18] S. O'Brien and J. L. Synge, Commun. Dublin Inst. Adv. Stud. A, no 9 (1952).

[19] R. Tabensky and A. H. Taub, Commun. Math. Phys. 29 (1973), 61.

[20] R. H. Gowdy, Ann. Phys. (N.Y.) 83 (1974), 203.

[21] R. H. Gowdy, J. Math. Phys. 16 (1975), 224.

[22] D. W. Kitchingham, Class. Quantum Grav. 1 (1984), 677; 3 (1986), 133.

[23] J. Centrella, Astrophys. J. 241 (1980), 875.

[24] J. Centrella and R. A. Matzner, Phys. Rev. D 25 (1982), 930.

[25] B. L. Beers and A. J. Dragt, J. Math. Phys. 11 (1970), 2313.

[26] C. W. Misner, J. Math. Phys. 4 (1963), 924. 
Figure 1: The embedding diagram of the section $Y=0$ (or $\phi=0$ and $\pi$ ) through the 3-hyperboloid (3.3) in a 3-dimensional Minkowski space. The 2-planes given by $z=$ $z_{0}=$ const., $(x, y) \in \mathbb{R}^{2}$ (or $(X, Y) \in \mathbb{R}^{2}$ ) are illustrated as parabolae along which $x, y$ vary.

Figure 2: The embedding diagram of the section $Y=0$ (or $\phi=0$ and $\pi$ ) through the 3-hyperboloid (3.3) in a 3-dimensional Minkowski space. The cylindrical 2-surfaces given by $\rho=\rho_{0}=$ const., $y \in(-\infty,+\infty), \phi \in[0,2 \pi)$ are illustrated in two hyperbolae representing two generators of the cylinder along which the coordinate $y$ varies. The axis of the cylinder given by $\rho=0$ is also indicated.

Figure 3: A space-time diagram illustrating the collision of cylindrical gravitational waves in a spatially flat or open FRW universe. Region I is the FRW background in cylindrical coordinates (5.5) or (5.7). Region II contains an outgoing cylindrical wave, region III contains an incoming cylindrical wave, and region IV is the interaction region following their collision. The space-time is only singular on the initial hypersurface $\eta=0$, and on the axis $\rho=0$.

Figure 4: Toroidal waves propagating into a closed FRW background. The figure shows two sections through the toroid as in figures 6 and 7 in the Appendix. The unshaded regions represent the exact FRW model, with the wave region illustrated schematically by the shaded areas. The singular circular axis $\zeta=0$ within the wave region is also indicated.

Figure 5: This space-time diagram illustrates the propagation of a gravitational wave into a closed FRW universe. The coordinates $\delta$ and $\sigma$ are suppressed. The shaded region contains the gravitational wave. The null wavefront is toroidal as illustrated in figure 4 . It starts at a line at the initial singularity $\eta=0$ and converges to another line at the final singularity $\eta=\pi / 2$. There is also a curvature singularity on the initial axis $\zeta=0$.

Figure 6: A section $Y=0(\phi=0$ and $\pi)$ through the 3 -sphere of radius $R_{0}$ showing circular cuts $A$ and $B$ through the toroidal surface. The circles $S, A$ and $B$ have radius $\kappa R_{0}$.

Figure 7: A section $Z=0(\theta=\pi / 2)$ through the 3 -sphere of radius $R_{0}$ showing circular cuts $C$ and $D$ through the toroidal surface. The points $a, a^{\prime}$ and $b, b^{\prime}$ are on the circles $A$ and $B$ respectively, indicated in figure 6 .

Figure 8: The entire spatial geometry of the closed FRW model at a given time is represented by two solid spheres. Points on the surface of upper hemisphere on the left on which $\sigma=0$ must be identified with points on the surface of the upper hemisphere on the right on which $\sigma=2 \pi$. Points on the surfaces of both lower hemispheres on which $\sigma=\pi$ must also be identified. Within each sphere, the segments are indicated which arise from the section $\delta=0$ and $\pi$ through the spheres. They represent the stereographic projections of the circles $A$ and $B$ in figure 6 onto the $(W, X)$ plane. 